\title{
Biopsy Specimen Size
}

National Cancer Institute

\section{Source}

National Cancer Institute. Biopsy Specimen Size. NCI Thesaurus. Code C139747.

The physical dimensions of a biopsy specimen. 